\title{
L’économie de la Fédération de Russie : 20 ans de transition au capitalisme (1991-2011)
}

Michel Roche, Ph. D.

Université du Québec à Chicoutimi

\section{INTRODUCTION}

En 1990, suite à l'échec de la perestroïka, les dirigeants de l'Union soviétique et de la future Russie indépendante décidaient de faire transiter leur pays vers l'économie de marché capitaliste. Vingt ans plus tard, la Russie est devenue méconnaissable. Dans cet article, nous tâcherons de mettre en exergue les changements les plus fondamentaux qui ont affecté l'économie du pays entre 1991 et 2011.

\section{LA TRANSITION : UN PROCESSUS DE TRANSFORMATIONS}

\section{La transition au capitalisme en Russie s'est distinguée par sa rapidité et sa brutalité. [...] Le passage à l'économie de marché était comparé à un "Big Bang ", un geste audacieux vers ce qui apparaissait comme l'unique voie d'avenir.}

La transition au capitalisme en Russie s'est distinguée par sa rapidité et sa brutalité. Les dirigeants russes, fortement influencés par le FMI et les dirigeants du G7, soutenaient qu'on ne pouvait franchir un gouffre en faisant deux bonds. Le passage à l'économie de marché était comparé à un «Big Bang », un geste audacieux vers ce qui apparaissait comme l'unique voie d'avenir. Cet empressement s'expliquait par des considérations non seulement économiques - la situation d'urgence causée par l'effondrement du système soviétique ${ }^{\mathrm{a}}$ - mais aussi politiques : la volonté de rendre la chute du «communisme » irréversible ${ }^{1}$. Il s'est imposé comme allant de soi dans les plus hautes sphères du pouvoir tout en répondant aux vœux exprimés par les dirigeants $\mathrm{du}$ G7 et des grandes organisations financières internationales. L'économiste Aleksandr Bouzgaline a expliqué le processus en se servant d'une métaphore intéressante. Imaginons le passage radical d'un système de transport public, conçu pour servir une grande population, à un système de transport privé et individuel : les infrastructures routières ne sont pas en place, il n'y a pas encore de panneaux de circulation réglementant la vitesse, les arrêts aux intersections, personne n'a appris à conduire, les pompes à essence sont insuffisantes de même que les stationnements, etc. L'économie soviétique était constituée d'entreprises publiques, la production et la distribution relevaient de la planification centralisée. Rien ne l'avait préparée au capitalisme.

La transition a été conçue en vertu du « consensus de Washington», fondé sur les principes du néolibéralisme, selon lesquels le marché et l'entreprise privée doivent souffrir le moins de contraintes possible, alors que le rôle de l'État doit demeurer confiné à la sécurité nationale, à l'élaboration des lois et à assurer la stabilité.

La transition a été conçue en vertu du " consensus de Washington ", fondé sur les principes du néolibéralisme, selon lesquels le marché et l'entreprise privée doivent souffrir le moins de contraintes possible.

\subsection{Transformation macroéconomique : la stabilisation}

La stabilisation macroéconomique avait pour objectif de permettre aux lois du marché de jouer leur rôle pour mettre fin aux pénuries par la "vérité des prix », en créant un cadre concur- 
rentiel. Ce processus impliquait notamment une politique de taux d'intérêt élevés destinés à combattre l'inflation, une déréglementation maximale, une monnaie convertible en fonction du marché, une réduction draconienne du déficit budgétaire, la suppression des subventions aux entreprises publiques, etc. La mesure la plus spectaculaire a été la libéralisation des prix, le 2 janvier 1992, qui a entraîné un taux d'inflation de plus de $2300 \%$ au cours de cette année. Les économistes néolibéraux comptaient sur l'offre et la demande pour stabiliser les prix. Or, les monopoles n'ayant pas été préalablement démantelés, les hausses de prix devenaient un moyen facile pour accroître les profits, sans pour autant mettre fin à la chute de la production industrielle et agricole. En effet, de 1992 à 1998, la chute de la production a dépassé celle qu'a subie l'URSS au cours de la Seconde Guerre mondiale. Les investissements se sont littéralement effondrés. Les ressources consacrées à la recherche et au développement ont régressé de $90 \%$.

Les salaires n'ayant pas progressé au même rythme que l'inflation, le niveau de vie a connu une baisse dramatique. Les pères de la thérapie de choc, dont le premier ministre en exercice Egor Gaïdar, prévoyaient une embellie après six mois de traitement. Ce n'est qu'en 1997 que les principaux objectifs de la stabilisation macroéconomique étaient atteints, alors que l'inflation ne dépassait plus $15 \%$ annuellement (sauf en 1998 et 1999). Les coûts sociaux se sont toutefois avérés prohibitifs. Nous y reviendrons plus loin.

La libéralisation du commerce faisait également partie des mesures proposées dans le cadre de la thérapie de choc. Une telle mesure impliquait la fin du monopole du commerce extérieur exercé par l'État, la convertibilité du rouble, l'alignement des prix intérieurs sur les prix à l'exportation.

La libéralisation du commerce faisait également partie des mesures proposées dans le cadre de la thérapie de choc. Une telle mesure impliquait la fin du monopole du commerce extérieur exercé par l'État, la convertibilité du rouble, l'alignement des prix intérieurs sur les prix à l'expor- tation. Dans un premier temps, les barrières tarifaires ont été réduites à zéro. Mais il en a résulté une véritable invasion de produits étrangers, au détriment de la production locale. Par la suite, le gouvernement russe a dû réinstaurer des droits de douane, mais à un niveau souvent bien inférieur à ce qui est en vigueur dans les économies dites « émergentes».

\subsection{Les innovations structurelles axées sur la privatisation}

Dans le cas de la Russie, la croyance partagée autant par les institutions financières

internationales que par le gouvernement russe de l'époque faisait de la privatisation de l'économie une panacée pour rendre l'économie du pays concurrentielle.

Les réformes structurelles se distinguent de la stabilisation macroéconomique par leur inscription dans la durée. Elles doivent assurer la croissance économique à long terme et non simplement répondre à une situation d'urgence. Dans le cas de la Russie, la croyance partagée autant par les institutions financières internationales que par le gouvernement russe de l'époque faisait de la privatisation de l'économie une panacée pour rendre l'économie du pays concurrentielle. Les monopoles étatiques étaient considérés inefficaces, trop lourds à gérer et générateurs de gaspillages. La Russie étant particulièrement bien pourvue en ressources de toutes sortes, notamment en matières premières et sources d'énergie, certains membres de la haute fonction publique, des cercles dirigeants, de l'armée, sans compter les entreprises étrangères, voyaient dans la transformation du pays l'occasion de mettre la main sur des richesses qui, jusque là, avaient échappé à l'accumulation du capital. Ils seront d'ardents promoteurs de la privatisation.

La privatisation touchait toutes les entreprises du pays, des plus petites, tels les cafés et les restaurants, jusqu'aux grands monopoles industrielles, les mines, la finance, etc. Si une entreprise aussi vaste que la privatisation de l'économie russe constituait un immense défi administratif et technique, les considérations 
politiques présentaient des difficultés plus complexes. La privatisation signifiait, concrètement, que les richesses produites collectivement depuis les années 1920 allaient passer entre les mains d'une minorité. Mais sur quelles bases cette minorité serait-elle désignée? Les travailleurs voyaient d'un mauvais œil l'arrivée de nouveaux patrons soucieux d'accroître la rentabilité au moyen de restructurations qui risquaient d'aboutir à des pertes d'emploi et à la mise à l'écart de la gestion paternaliste qui avait caractérisé la période soviétique dans un contexte de pénurie de main-d'œuvre. Les directeurs d'entreprise craignaient également pour leurs postes.

Aussi, les pressions ont été fortes au point où le gouvernement a dû reculer en acceptant une formule permettant aux travailleurs et directeurs, dans les grandes entreprises, de posséder jusqu'à $51 \%$ des actions, quoique sur une base individuelle, la propriété collective étant formellement interdite par la loi. De même, le Comité de gestion de la propriété de l'État (GKI), dans un souci de rendre le processus relativement populaire, a procédé à la distribution de bons de privatisation à chaque citoyen du pays, y compris les nouveau-nés. En dépit de ces mesures, la propriété des entreprises s'est rapidement concentrée entre les mains de quelques individus ${ }^{\mathrm{b}}$, en majorité ceux qui étaient proches du pouvoir ou de la haute direction des principaux ministères. D'ailleurs, l'épargne accumulée par des millions de Soviétiques a été anéantie par l'hyperinflation engendrée par la libéralisation des prix, pavant la voie aux seuls détenteurs de liquidités ${ }^{2}$. «Nous n'avons jamais eu pour but de faire de chaque citoyen un propriétaire ${ }^{3} »$, déclarait, en mars 1994 , Anatoli Tchoubaïs, dirigeant du GKI et père de la privatisation russe. En 1996, le taux de privatisation des entreprises russes atteignait $70 \%$. Il faut se rappeler qu'en dix ans, Margaret Thatcher avait privatisé $5 \%$ de l'économie britannique.

La propriété des entreprises s'est rapidement concentrée entre les mains de quelques individus, en majorité ceux qui étaient proches du pouvoir ou de la haute direction des principaux ministères.
En 1996, l’État russe se trouvant en manque de liquidités, des joyaux de l'économie russe ont été vendus pour une fraction de leur valeur marchande, principalement dans l’industrie du charbon, du gaz et du pétrole.

La privatisation russe a été marquée par la corruption à grande échelle. Les ventes aux enchères avaient lieu dans les banques privées, dont l'accès était souvent restreint, les élus chargés de la vente des actifs municipaux (et de déterminer les prix) formaient des sociétés privées qui les achetaient. Des ministères se sont eux-mêmes transformés en sociétés privées, notamment le ministère du Gaz, devenu Gazprom. L'accumulation de richesses ayant été formellement illégale sous le régime soviétique, les éléments mafieux ont pu mettre la main sur un grand nombre d'entreprises qui leur ont permis de blanchir leur argent. En 1996, l'État russe se trouvant en manque de liquidités, des joyaux de l'économie russe ont été vendus pour une fraction de leur valeur marchande, principalement dans l'industrie du charbon, du gaz et du pétrole. Ce sont surtout les banques privées qui en ont profité et ce, d'autant plus facilement qu'elles procédaient elles-mêmes à la vente aux enchères. Au total, on a calculé que les 500 plus importantes entreprises de Russie, évaluées à 200 milliards de dollars, ont été cédées pour environ 7,2 milliards, soit 3,6\% de leur valeur.

De sceptique qu'elle était au début, la majorité de la population est devenue hostile à la propriété privée des grandes entreprises. On a rapidement associé la privatisation à la catastrophe économique et sociale qui l'a accompagnée.

De sceptique qu'elle était au début, la majorité de la population est devenue hostile à la propriété privée des grandes entreprises. On a rapidement associé la privatisation à la catastrophe économique et sociale qui l'a accompagnée. De 1991 à 1995, le PIB a reculé d'environ $40 \%$, la production industrielle de presque $50 \%$. Ce n'est qu'en 1997 que le PIB connaissait une croissance positive, à $1 \%$. En 2001, le PIB atteignait les deux tiers de celui de $1988^{4}$. 
Tableau 1 : Taux de croissance du PIB et de l'inflation (1991-1999)

\begin{tabular}{|c|c|c|}
\hline & $\begin{array}{c}\text { Taux de croissance du PIB } \\
\text { (en prix constants du marché (8)) }\end{array}$ & Taux d'inflation \\
\hline 1991 & -5.0 & 138.0 \\
\hline 1992 & -14.5 & 2323.0 \\
\hline 1993 & -8.7 & 844.0 \\
\hline 1994 & -12.6 & 202.0 \\
\hline 1995 & -4.3 & 131.0 \\
\hline 1996 & -6.0 & 21.8 \\
\hline 1997 & 0.4 & 11.3 \\
\hline 1998 & -11.6 & 84.4 \\
\hline 1999 & 3.2 & 36.5 \\
\hline
\end{tabular}

Source: Rapports de presse du Service des Statistiques de l'État russe, cité par Remington (3édition), p. 199.

Encore aujourd'hui, la majorité des Russes considèrent que les grandes entreprises devraient demeurer propriété de l'État. Ce sentiment de méfiance sinon d'hostilité à l'égard de la méthode employée pour privatiser l'économie et de ses résultats explique en grande partie la peur ressentie par les élites financières et politiques de la Russie à l'égard de la démocratie, dont le plein déploiement risquerait de remettre en cause l'ordre établi.

\section{IMPACT DE LA TRANSITION SUR LA PLACE DE L'ÉCONOMIE RUSSE DANS LE MONDE}

\subsection{Périphérisation}

\section{L'URSS, presque totalement isolée du reste du monde entre 1917 et la Deuxième Guerre mondiale, a dû développer l'économie dans tous les domaines dans un cadre quasi autarcique.}

L'URSS, presque totalement isolée du reste du monde entre 1917 et la Deuxième Guerre mondiale, a dû développer l'économie dans tous les domaines dans un cadre quasi autarcique. Après la guerre, la soviétisation de l'Europe centrale et orientale et la victoire des communistes en Chine lui ont permis de briser cet isolement. Cependant, ces pays ne se trouvaient nullement dans une situation où ils auraient pu venir en aide à l'URSS pour des transferts technologiques ou autres. L'Union soviétique, malgré des avancées certaines dans plusieurs domaines, n'a jamais pu atteindre le niveau des pays capitalistes développés. Ses marchandises trouvaient difficilement preneurs à l'extérieur de la zone économique rassemblant l'URSS et ses alliés, le Comecon. Néanmoins, elle produisait ses propres avions, son propre matériel militaire, ses fusées spatiales, ses voitures; elle formait sa communauté scientifique, l'une des plus importantes du monde; sa main-d'œuvre qualifiée était nombreuse. Bref, l'URSS, bien que différente des sociétés industrielles développées et en dépit de toutes ses difficultés, ne partageait aucunement les caractéristiques des économies périphériques du tiers-monde.

La transition a modifié complètement la situation. Après l'effondrement des régimes prosoviétiques en 1989, les partenaires européens de l'URSS se sont tournés vers l'Union européenne. Dès 1991, le commerce entre les anciens membres du Comecon a reculé de $60 \%$. La part des échanges avec les pays de l'OCDE est passée de $25 \%$ à $60 \%$, entre 1989 et 1992 . L'éclatement de l'URSS amenait également les quatorze républiques non-russes à réorienter leur commerce. Les républiques d'Asie centrale ont redirigé leur commerce vers le Moyen-Orient, le Pakistan, l'Iran. La Russie indépendante, qui formait jusque là le cœur d'un système, commençait à se périphériser en se tournant vers des puissances économiques plus avancées qu'elle $\mathrm{du}$ point de vue des infrastructures, du savoir- 
faire, de la compétitivité, de la productivité du travail et du niveau de vie.

\section{L’arrivée de la Russie dans l'économie mondiale dominée par les puissances \\ économiques les plus développées, célébrée avec enthousiasme du point de vue politique et idéologique, ne s'est pas traduite de façon aussi accueillante en matière économique. Derrière l'allié nouvellement converti au capitalisme se dissimule un concurrent potentiel.}

Dans l'immédiat, seules ses matières premières et produits énergétiques lui offraient un avantage comparatif par rapport à ses partenaires. Le territoire russe contient d'importants gisements d'uranium, d'or, de diamants. Il est en grande partie couvert d'immenses forêts. Son sous-sol contiendrait respectivement $13 \%$ et $26 \%$ des réserves mondiales de pétrole et de gaz naturel ${ }^{\mathrm{c}}$.

Il convient ici de souligner que l'arrivée de la Russie dans l'économie mondiale dominée par les puissances économiques les plus développées, célébrée avec enthousiasme du point de vue politique et idéologique, ne s'est pas traduite de façon aussi accueillante en matière économique. Derrière l'allié nouvellement converti au capitalisme se dissimule un concurrent potentiel. Contrairement à ce qui s'est produit pour les pays d'Europe centrale et orientale ou pour la Chine, les conditions imposées à la Russie ont été particulièrement contraignantes. L'adhésion au FMI et à la Banque mondiale en 1992 a été assortie de conditions beaucoup plus sévères pour elle que pour la Pologne de Jaruzelski ou la Roumanie de Ceausescu ${ }^{5}$.

Depuis 1995, elle tente d'adhérer à l'OMC, mais sans succès jusqu'ici ${ }^{6}$. Ce n'est pas sans raison que les dirigeants russes critiquent leurs partenaires états-uniens et européens et les soupçonnent de vouloir confiner la Russie au rôle de simple pourvoyeur de ressources naturelles et énergétiques. En dépit du discours libreéchangiste dominant, les barrières restent encore relativement élevées pour les produits en provenance de la Russie. Le pétrole et le gaz demeurent les marchandises les plus faciles à écouler sur les marchés mondiaux. À l'inverse, la Russie cherche à protéger certains secteurs de son économie de la concurrence étrangère. À titre d'exemple, l'agriculture russe survivrait difficilement face aux produits agricoles européens, largement subventionnés.

\subsection{Commerce extérieur}

De la fin de l'époque soviétique à nos jours, le panier des exportations russes vers les pays capitalistes développés n'a guère changé. L'URSS exportait vers l'Europe de l'Ouest des produits énergétiques. Cependant, l'Europe de l'Ouest ne comptait que pour 20 à $25 \%$ des exportations de l'URSS. Au total, les deux tiers des exportations soviétiques étaient constitués de produits à faible valeur ajoutée, le pétrole comptant pour plus de la moitié 7 .

L'URSS importait surtout des équipements, mais en provenance de la RDA, de la Tchécoslovaquie et de la Hongrie. Néanmoins, ces données ne suffisent pas pour conclure à une économie de type périphérique. Les trois quarts du commerce extérieur de l'URSS échappaient aux lois du marché: le commerce intraComecon demeurait de loin le plus important pour ses membres, dont l'URSS, et était dicté par les besoins de l'économie planifiée. Par ailleurs, dans une relation de type périphérique, les pays de la périphérie n'ont que très peu de rapports entre eux, étant tournés vers un Centre ${ }^{8}$.

Une fraction non négligeable des exportations de l'URSS était constituée de marchandises à haute valeur ajoutée : les armes. Ce marché a reculé considérablement au cours de la thérapie de choc. Ses anciens alliés du Comecon sont devenus membres de l'OTAN et achètent des armes en provenance des États-Unis et de leurs alliés.

Il reste qu'en seulement deux ans, l'espace économique que constituait le Comecon et qui donnait à ce bloc une autonomie relative par rapport au marché mondial et aux pays capitalistes développés a été détruit. L'URSS se périphérise dans la mesure où sa dépendance envers l'Union européenne s'accroît. Cette périphérisation la ramène vers le type de relation qu'entretenait la Russie tsariste avec cette région du monde avant la Révolution de 1917. 


\section{IMPACT DE LA TRANSITION AU CAPITALISME SUR LE NIVEAU DE VIE}

\subsection{Les revenus et la distribution de la richesse}

Les ouvriers comptent parmi les catégories ayant le plus souffert de la transition. Si le régime soviétique les privait des libertés politiques les plus fondamentales, il se souciait toutefois de leur sécurité économique, surtout après la mort de Staline, quand a été abolie la terreur de masse. Même s'il s'agissait sans doute d'un moyen pour entretenir leur passivité, cette gestion paternaliste de l'État et des directions d'entreprise provenait également de la nature même de la planification centralisée : la production n'était pas motivée par la recherche du profit mais par l'intérêt de la chaîne de commandement d'atteindre les indices de production décrétés par les autorités centrales ${ }^{9}$. Ainsi, les entreprises cherchaient à attirer les travailleurs en leur offrant les meilleures conditions de travail que leur permettaient les moyens à leur disposition. Les salaires progressaient sur une base régulière, la discipline au travail n'était pas trop stricte, la production fonctionnait au ralenti pendant certaines périodes. De cette situation a résulté un sur-emploi qui a abouti à une pénurie de main-d'œuvre, à l'échelle du pays.
Au moment où l'URSS/Russie entreprenait sa transition au capitalisme, le taux de chômage se situait à zéro pour cent. En 2000, il frôlait les $10 \%$, d'après les statistiques officielles qui sous-estiment gravement le phénomène. La thérapie de choc et l'implosion de l'URSS ont entraîné une chute dramatique du niveau de vie. Selon la Banque mondiale, en 1988, 1,5\% de la population soviétique vivait dans la pauvreté; au milieu de l'année 1993, ce pourcentage serait passé dans une fourchette de 39 à $49 \%{ }^{10}$. Les salaires réels ont diminué de moitié au cours de la première année de la thérapie de choc. Mais cela ne suffit pas à expliquer la baisse du niveau de vie général, qui ne dépendait pas que du seul salaire. Dans les années 1980, sous le régime soviétique, pour chaque rouble versé en salaire dans les entreprises, l'État ajoutait 69 kopeks sous diverses formes : services sociaux, biens de consommation subventionnés, transports publics à bon marché, etc. Avec la thérapie de choc, $87 \%$ de ces dépenses ont été transférées aux gouvernements locaux, qui n'avaient pas les moyens de les verser ${ }^{11}$.

Tableau 2 : Statistiques sur le chômage en Russie (1992-2000)

\begin{tabular}{|c|c|c|c|}
\hline Année & 1992 & 1995 & 2000 \\
\hline $\begin{array}{c}\text { Nombre de } \\
\text { chômeurs } \\
\text { (en milliers) }\end{array}$ & 3889 & 6684 & 7059 \\
\hline $\begin{array}{c}\text { Taux de } \\
\text { chômage }\end{array}$ & 5,2 & 9,5 & 9,8 \\
\hline
\end{tabular}

Source : Федеральная служба государственой статистики, Россия в изиррах, Официальное издание, (Service fédéral des statistiques d'État, La Russie en chiffres, publication officielle), édition 2009, p. 91 Sur le taux de chômage : p. 92.

La vaste majorité de la population a donc perdu la sécurité économique en vigueur jusque là, sans obtenir de compensations d'ordre monétaire en retour. Vers la fin de l'époque soviétique, une classe moyenne urbaine, bien éduquée, a servi d'appui à la pérestroïka. Elle a été considérablement réduite et appauvrie par la thérapie de choc. En 2010, le revenu moyen n'avait toujours pas rattrapé le niveau de 1990. 
Tableau 3 : Nombre et pourcentage d'individus pauvres, 1991-2000, suivant le critère de revenus inférieurs au minimum de subsistance

\begin{tabular}{|l|c|c|c|c|c|c|c|c|c|c|}
\hline & 1991 & 1992 & 1993 & 1994 & 1995 & 1996 & 1997 & 1998 & 1999 & 2000 \\
\hline $\begin{array}{l}\text { Nombre, } \\
\text { en millions }\end{array}$ & $\begin{array}{c}11 \\
(\mathrm{BM})\end{array}$ & 49,7 & $\begin{array}{c}46 \\
(\mathrm{BM})\end{array}$ & 33,3 & 35,5 & 32,0 & 31,0 & 34,2 & 41,6 & 42,3 \\
\hline $\begin{array}{l}\text { En \% de la } \\
\text { population }\end{array}$ & 7 & 33,5 & $\begin{array}{c}32 \\
(\mathrm{BM})\end{array}$ & 22,4 & 24,7 & 22,1 & 21,1 & 23,3 & 28,4 & 29,1 \\
\hline
\end{tabular}

Sources : Pour les années 1992, 1994, 1996 et suivantes : Goskomstat, 1999 et 2002. Pour 1991 et 1993, Banque Mondiale (BM), 2000. Pour 1995, chiffres fournis par Lilia Ovčarova, Institut des problèmes socio-économiques de la population, Moscou. Tableau tiré de Cécile Lefèvre, «Enfance, famille et institutions en Russie au cours des années 1990 : un aperçu des évolutions récentes», Revue d'études comparatives EstOuest, 2003, vol. 34, no. 2, p. 85.

Par ailleurs, l'indice du revenu moyen dissimule de très fortes inégalités sociales. Les inégalités existaient certes sous le régime soviétique, mais c'était sans commune mesure avec la situation actuelle. En 1990, le rapport entre le premier décile et le dernier était de 1 à 4; en 1998, il se situait à 1 à 13 et même 1 à 30 . Aux inégalités sociales s'ajoutent des inégalités régionales. Une partie disproportionnée de la richesse du pays se concentre à Moscou, à SaintPétersbourg et dans quelques villes et régions particulièrement prospères grâce à l'industrie pétrolière.

\section{Économie périphérique et société non périphérique (population éduquée et urbanisée, main-d'œuvre qualifiée) forment l'une des contradictions les plus frappantes de la Russie actuelle. La désindustrialisation a frappé toutes les régions, sans que le secteur tertiaire ne soit en mesure de prendre le relais au même rythme.}

Si l'économie russe affiche un portrait comportant de nombreuses similitudes avec la périphérie de l'économie mondiale, il en est tout autrement de la société. Économie périphérique et société non périphérique (population éduquée et urbanisée, main-d'œuvre qualifiée) forment l'une des contradictions les plus frappantes de la Russie actuelle $^{\mathrm{d}}$. La désindustrialisation a frappé toutes les régions, sans que le secteur tertiaire ne soit en mesure de prendre le relais au même rythme. Cette particularité explique qu'une partie significative de la population russe occupe un emploi exigeant des qualifications inférieures eu égard à la formation reçue. En
2007, la Russie dépasse de loin la moyenne des pays de l'OCDE, avec près de $55 \%$ de la population ayant obtenu au minimum une éducation tertiaire. Aux États-Unis, ce chiffre atteint à peine $40 \%{ }^{12}$. La pérestroïka avait notamment pour objectif de réduire l'écart entre les technologies utilisées et le niveau de formation de la main-d'œuvre. Pour des millions de Russes, ce problème s'est amplifié.

La santé publique a connu une détérioration sans précédent. Des maladies éradiquées sous le régime soviétique sont réapparues. L'espérance de vie des hommes, de 64 ans en 1990, reculait à 55 ans en 1998; $40 \%$ des jeunes garçons de 16 ans n'atteindront pas l'âge de 60 ans. Le pays fait par ailleurs face à une crise démographique, commencée sous le régime soviétique mais brusquement amplifiée avec la thérapie de choc: le taux de natalité a chuté de $6 \%$ entre 1989 et 1997, alors que le taux de mortalité a grimpé de 3,5\% au cours de la même période. En conséquence, en 2000, la Russie comptait deux décès pour une seule naissance. Entre 1989 et 2000, le nombre d'enfants de zéro à neuf ans est passé de 24 à 15 millions, ce qui représente une diminution de $37 \%$. Les statistiques de l'année 2000 révélaient un million de naissances de moins qu'en $1990^{13}$. Le taux de scolarisation connaissait lui aussi une baisse marquée, de $8 \%$. Les enfants abandonnés, nombreux au cours des deux guerres mondiales et de la guerre civile mais totalement pris en charge sous le régime soviétique, ont refait leur apparition. En somme, c'est comme si la Russie en transition avait été ravagée par une guerre. 


\section{ENJEUX ACTUELS : DES INNOVATIONS À CONSOLIDER AVEC PRÉCAUTION}

La Russie a renoué avec la croissance après la crise financière de 1998 et l'inflation est beaucoup mieux contrôlée (voir tableau IV) Depuis, le budget de l'État s'est amélioré et le niveau de vie a progressé. Néanmoins, l'économie russe doit encore faire face à des problèmes majeurs.

Tableau 4 : Taux de croissance du PIB et taux d'inflation

\begin{tabular}{|l|c|c|c|c|c|c|c|c|c|c|c|}
\hline Année & 2000 & 2001 & 2002 & 2003 & 2004 & 2005 & 2006 & 2007 & 2008 & 2009 & $2010^{\star}$ \\
\hline $\begin{array}{l}\text { Taux de } \\
\text { croissance } \\
\text { du PIB (1) }\end{array}$ & 9.0 & 5.0 & 4.7 & 7.3 & 7.1 & 6.4 & 6.7 & 8.1 & 5.6 & -8.7 & 4.9 \\
\hline $\begin{array}{l}\text { Taux } \\
\text { d'inflation (2) }\end{array}$ & 20,2 & 18,6 & 15,1 & 12,0 & 11,7 & 10,9 & 9,0 & 11,9 & 13,3 & 8,8 & 7,5 \\
\hline
\end{tabular}

(1) Source : OCDE;. (2) Source : Comité d'État des statistiques (Goskomstat); * prévision

\subsection{La dépendance}

La dépendance de l'économie russe à

l'égard des ressources naturelles et produits énergétiques figure parmi les plus importants enjeux auxquels le gouvernement doit faire face. Entre 2001 et 2004, ce secteur a contribué à hauteur de $70 \%$ de la croissance industrielle.

La dépendance de l'économie russe à l'égard des ressources naturelles et produits énergétiques figure parmi les plus importants enjeux auxquels le gouvernement doit faire face. Entre 2001 et 2004 , ce secteur a contribué à hauteur de $70 \%$ de la croissance industrielle ${ }^{14}$. À elle seule, la production de pétrole et de gaz représente $60 \%$ des revenus du budget de l'État et explique la plus grande partie de la croissance de la production industrielle de 1999 à 2008. L'augmentation des exportations et du prix du baril au cours de cette période a permis à la Russie de rembourser la quasi-totalité de sa dette ${ }^{\mathrm{e}}$ et de constituer la troisième plus grande réserve de devises du monde. En même temps, comme l'a démontré la crise financière de 2008-2009, elle expose à la face du monde la vulnérabilité de la Russie à toute baisse du prix du pétrole, du gaz naturel et des autres matières premières ${ }^{f}$. Par ailleurs, selon le vice-ministre des ressources naturelles, Alexeï Varlamov, les ressources énergétiques de la Russie pourraient être épui- sées dans 10 ou 15 ans $^{15}$. Ce pronostic peut sembler arbitraire, mais témoigne néanmoins de la nécessité d'asseoir l'économie russe sur des assises plus solides au cours des décennies à venir.

Le président Medvedev s'est d'ailleurs donné pour objectif de moderniser et de diversifier l'économie. En mai 2010, pour bien faire sentir l'urgence d'agir, il prévenait un auditoire composé de hauts fonctionnaires du pays, que « la Russie va s'effondrer, à moins que sa structure sociale et son économie ne soient modernisées ${ }^{16} »$. Les prix élevés du pétrole auraient pu dégager une partie des sommes nécessaires à cette restructuration, mais ce ne fut pas le cas. Les capitaux se dirigent vers les secteurs où les perspectives d'accumulation demeurent les plus élevées, ce qui ne favorise guère l'industrie russe, à l'exception des matières premières et des produits semi-finis, tel l'aluminium. Cette situation incite les entrepreneurs russes les plus fortunés à investir leur argent à l'étranger. Le groupe financier suisse UBS et la compagnie Campden Research ont mené une enquête auprès de 25 entrepreneurs russe dont la fortune s'établit dans une fourchette allant de 100 à 500 millions de dollars. Cette enquête révèle que $84 \%$ d'entre eux ont placé leur argent à l'extérieur de la Russie, principalement à Chypre, en Suisse et aux Îles Vierges ${ }^{17}$. 
En 2010, le gouvernement russe projette l'aménagement d'une «cité de

l'innovation", Skolkovo, en banlieue de Moscou, qui ferait office de «Silicon

Valley» russe.

La dépendance de l'économie russe de la rente pétrolière et gazière nuit de plus en plus à l'industrie: les marchandises produites par l'industrie russe coûtent de plus en plus cher, du fait de la montée du rouble, causée par la hausse des prix des matières premières et de l'énergie : la valeur des exportations de ce secteur est passée de 55,5 milliards de dollars en 2000 à 326 milliards en $2008^{18}$. Cette situation a pour effet de rendre l'industrie russe encore moins compétitive, ce que les économistes appellent la «maladie hollandaise»: les exportations de matières premières font monter la valeur du rouble, faisant ainsi augmenter le prix des autres marchandises produites par l'économie russe. Pour remédier à cela, il faudrait investir davantage dans la R\&D, mais la Russie se trouve loin des pays capitalistes développés à cet égard. En 2008 , le pays ne consacrait que $1,03 \%$ de son PIB à la recherche et au développement, contre environ $2,7 \%$ aux États-Unis et $3,5 \%$ en Finlande. En 1990, l'URSS allouait plus de $2 \%$ de sa richesse nationale à ce domaine. Avec la thérapie de choc, ce pourcentage est descendu aussi bas que $0,7 \%$. Par ailleurs, la plus grande partie des dépenses est assumée par l'État et destinée à des fins militaires. En 2010, le gouvernement russe projette l'aménagement d'une " cité de l'innovation », Skolkovo, en banlieue de Moscou, qui ferait office de «Silicon Valley $\gg$ russe $^{\mathrm{g}}$.

Tableau 5 : Dépenses en R\&D, en pourcentage du PIB (1990-2008)

\begin{tabular}{|l|l|l|l|l|l|l|l|l|l|l|l|}
\hline 1990 & 1992 & 1999 & 2000 & 2001 & 2002 & 2003 & 2004 & 2005 & 2006 & 2007 & 2008 \\
\hline 2,03 & 0,74 & 1,0 & 1,05 & 1,18 & 1,25 & 1,28 & 1,15 & 1,07 & 1,07 & 1,12 & 1,03 \\
\hline
\end{tabular}

Source : OECD Factbook 2010: Economic, Environmental and Social Statistics - ISBN 92-64-08356-1 - C OECD 2010

En attendant, la compétitivité des entreprises russes est redevable essentiellement à des coûts de production moins élevés, mais cet avantage se perd avec la montée de la Chine et de l'Inde. En Russie, la productivité est du même ordre qu'en Inde et en Chine, mais les coûts de maind'œuvre y demeurent plus élevés. Elle est nettement inférieure à celle des pays capitalistes avancés. À titre d'exemple, le constructeur automobile russe Avtovaz produit 736000 voitures avec 104000 travailleurs, alors que l'entreprise française Renault en fabrique 2,43 millions avec 130000 travailleurs ${ }^{19}$. Au total, un travailleur d'Avtovaz produit 7,1 voitures annuellement contre 18,7 pour son collègue employé chez Renault. Ces chiffres témoignent du retard technologique de l'industrie russe, retard appelé à s'accroître si aucun effort véritable ne change la donne en matière de R\&D.
Pour procéder à la modernisation de l'économie russe, les dirigeants doivent aussi compter sur l'investissement étranger. La crise financière a toutefois eu pour effet de faire reculer le volume des investissements directs étrangers (IDE) en Russie, de 70 milliards de dollars en 2008 à 38,7 milliards en 2009.

Pour procéder à la modernisation de l'économie russe, les dirigeants doivent aussi compter sur l'investissement étranger. La crise financière a toutefois eu pour effet de faire reculer le volume des investissements directs étrangers (IDE) en Russie, de 70 milliards de dollars en 2008 à 38,7 milliards en 2009. D'un point de vue plus structurel, l'un des obstacles à l'investissement étranger réside dans le degré élevé de corruption, qui compte pour environ le tiers du PIB. D'après la liste établie par Transparency International, en 2010, la Russie figurait au $154^{\mathrm{e}}$ rang sur 178 . 
Jusqu'à présent, les efforts déclarés des gouvernements successifs n'ont produit aucun effet notable. À l'inverse, l'État utilise tous les moyens à sa disposition pour assurer la «paix sociale ». Le pouvoir politique demeure fortement concentré dans les mains de l'Exécutif, les médias électroniques font preuve d'une grande prudence pour éviter de contrarier les autorités, les manifestations, pourtant autorisées par la Constitution, se heurtent à des obstacles rendant leur organisation difficile. Dans un tel contexte, l'ordre établi apparaît encore relativement peu contesté, en dépit de l'augmentation sensible du nombre de grèves depuis 2007.

Le président Medvedev, fidèle au « consensus de Washington "), encourage la privatisation de ce qui demeure encore propriété de l'État. Sous Vladimir Poutine, le secteur contrôlé par l'État s'est sensiblement accru, passant de 24 à $40 \%$. Medvedev estime que la compétition demeure le meilleur moyen pour stimuler l'innovation ${ }^{20}$. Il a d'ailleurs signé un décret, en juin 2010, visant à réduire de $80 \%$ le nombre d'entreprises considérées "stratégiques » et bénéficiant donc, jusque là, du soutien de l'État.

\subsection{La démographie}

D’après la Banque mondiale, la Russie aura besoin de plus de 12 millions d'immigrants au cours des $\mathbf{2 0}$ prochaines années pour compenser la diminution de la population active de 800000 à 1,1 million annuellement, à partir de 2013.

La crise démographique ajoute aux difficultés actuelles et à venir de l'économie russe. Cette crise pourrait se traduire par une pénurie de main-d'œuvre et des charges sociales plus lourdes pour la population active. Les données les plus récentes font état d'un net ralentissement du déclin démographique, sinon d'une légère amélioration. Le premier trimestre de l'année 2010 a vu le nombre de naissances augmenter à 428336 - une hausse de près de 6500 par rapport à la même période en 2009 et le nombre de décès reculer à 515600 , soit une diminution d'environ $11000^{21}$. Néanmoins, le taux de croissance naturelle demeure négatif. D'après la Banque mondiale, la Russie aura besoin de plus de 12 millions d'immigrants au cours des 20 prochaines années pour compenser la diminution de la population active de 800000 à 1,1 million annuellement, à partir de $2013^{22}$.

Tableau 6 : Population de la Fédération de Russie (en millions d'habitants)

\begin{tabular}{|c|c|c|c|c|c|c|c|c|c|c|}
\hline 1992 & 1995 & 2000 & 2002 & 2003 & 2004 & 2005 & 2006 & 2007 & 2008 & $2010^{*}$ \\
\hline 148,6 & 148,3 & 146,3 & 145,2 & 144,2 & 143,5 & 142,8 & 142,2 & 142,0 & 141,9 & 141,2 \\
\hline
\end{tabular}

Source : Федеральная служба государственой статистики, Россия в цифрах, Официальное издание, (Service fédéral de statistiques d'État, La Russie en chiffres, publication officielle), édition 2009, p. 28. * Résultats préliminaires

L'un des membres du gouvernement russe a déjà pris position en faveur d'une augmentation de l'âge de la retraite, actuellement à 60 ans pour les hommes et 55 ans pour les femmes. Mais quelles en seraient les conséquences, dans un pays où l'espérance de vie des hommes n'atteint pas encore les 62 ans $^{\mathrm{h}}$ ?

\subsection{Les conditions de vie}

La situation sociale continue d'être alarmante pour une majorité de la population. D'après les données compilées par le PNUD, l'indice de développement humain ne s'est guère amélioré et ce, en dépit de la forte croissance des années
2000. De 0,821 qu'il était en 1990, l'indice a plongé à 0,777 en 1995 , pour remonter à 0,817 en 2009. Membre du G8, la Russie ne figure qu'au $71^{\mathrm{e}}$ rang, tout juste après l'Albanie ${ }^{23}$.

Les statistiques font état d'une amélioration réelle du niveau de vie depuis 1999. Mais il s'agit là de moyennes qui ne donnent qu'un aspect de la réalité. Moins de $0,2 \%$ de la population accapare plus de la moitié de la richesse du pays. La liste des milliardaires compilée par Forbes pour l'année 2007 comprenait 52 Russes (il n'y en avait aucun en 2000), dont 14 figuraient parmi les 100 personnes les plus 
riches du monde. On comptait alors davantage de milliardaires à Moscou qu'à New York alors que le salaire moyen brut à Moscou atteignait à peine le quart de celui de New York. Par ailleurs, la récession de 2009 a provoqué une détérioration des statistiques sur le niveau de vie. Une équipe de chercheurs de l'Université d'État - Hautes études en économie (SU-HSE) a pu démontrer que le revenu réel médian des ménages avait chuté de $6 \%$ - une première depuis 1998 - et que les dépenses moyennes des ménages avaient reculé de $12 \%{ }^{24}$.

Le taux de chômage a certes régressé, mais dans des proportions somme toute modestes, dans la mesure où les statistiques officielles reflètent adéquatement la réalité.
Alors que le ministère de la Santé et du Développement social situe le nombre de chômeurs à 6,5 millions en mai 2010 (7,5 \%), la méthode utilisée par l'Organisation internationale du travail (OIT) ferait monter ce chiffre à environ 8 millions. Mais selon Nikolaï Volguine, qui dirige le Département de la politique social et du travail de l'Académie de la Fonction publique russe, le taux de chômage réel incluant le chômage caché - atteindrait les $20 \%$, soit plus de 16 millions d'individus faisant partie de la population active ${ }^{25}$. Volguine tient compte $\mathrm{du}$ fait qu'un grand nombre de chômeurs ne s'inscrivent pas au programme d'assurancechômage.

Tableau 7 : Statistiques sur le chômage en Russie (2000 à 2008)

\begin{tabular}{|l|c|c|c|c|c|c|c|}
\hline Année & 2000 & 2003 & 2004 & 2005 & 2006 & 2007 & 2008 \\
\hline $\begin{array}{l}\text { Nombre de } \\
\text { chômeurs } \\
\text { (en milliers) }\end{array}$ & 7059 & 5683 & 5775 & 5208 & 4999 & 4246 & 5286 \\
\hline $\begin{array}{l}\text { Taux de } \\
\text { chômage }\end{array}$ & 9,8 & 7,8 & 7,9 & 7,1 & 6,7 & 5,7 & 7,0 \\
\hline
\end{tabular}

Source : Федеральная служба государственой статистики, Россия в цифрах, Официальное издание, (Service fédéral de statistiques d'État, La Russie en chiffres, publication officielle), édition 2009, p. 91 Sur le taux de chômage : p. 92. En 2009, le taux de chômage aurait grimper à 8,5\%, d'après Alexander Surinov, qui dirige le Service fédéral des Statistiques. Itar-Tass, 22 juin 2010.

\section{CONCLUSION}

\section{Au contraire, la Russie s’intègre à une économie mondiale déjà constituée et hiérarchisée. Elle subit la division du travail en vigueur. Les dirigeants cherchent naturellement à modifier cette hiérarchie, cette division du travail en fonction de ce qu'ils perçoivent comme étant l’intérêt de la Russie.}

Comme on l'a vu, la transition à l'économie capitaliste pose la question de la place que la Russie est appelée à jouer dans un monde qu'elle n'a pas façonné à sa manière et à son image, comme c'était le cas dans l'espace du «camp socialiste » qu'elle dominait. $\mathrm{Au}$ contraire, la Russie s'intègre à une économie mondiale déjà constituée et hiérarchisée. Elle subit la division du travail en vigueur. Les dirigeants cherchent naturellement à modifier cette hiérarchie, cette division du travail en fonction de ce qu'ils perçoivent comme étant l'intérêt de la Russie. Dans un premier temps, le gouvernement russe a joué la carte du néolibéralisme, tel que prôné par les grandes institutions internationales, contrôlées par le G7. La crise financière de 1998 a entraîné une rectification en cette matière, compte tenu des dégâts causés à l'industrie russe. Par la suite, la hausse des prix du pétrole et du gaz ont permis à la Russie de mettre de l'avant une politique plus nationaliste, impliquant un renforcement du rôle de l'État ${ }^{\mathrm{i}}$. La présidence de Vladimir Poutine et de son «successeur", Dmitri Medvedev, symbolise ce virage nationaliste, parfois plus symbolique que réel. N'empêche, le gouvernement russe, depuis le début de ce siècle, a su provoquer l'ire des États-Unis et de 
leurs proches alliés beaucoup plus souvent qu'à l'époque de Boris Eltsine. À ce titre, la Russie se comporte comme un pays capitaliste normal, qui cherche à protéger les intérêts de sa propre bourgeoisie. C'est ainsi, par exemple, qu'il faut comprendre les prétentions de ce pays sur la région du Pôle Nord, et non comme un nouvel épisode de la Guerre froide.

La Russie se comporte comme un pays capitaliste normal, qui cherche à protéger les intérêts de sa propre bourgeoisie. C'est ainsi, par exemple, qu'il faut comprendre les prétentions de ce pays sur la région du Pôle Nord, et non comme un nouvel épisode de la Guerre froide.

Il reste toutefois que dans ses tentatives pour s'intégrer à l'économie mondiale dans un sens plus conforme à ses intérêts, la Russie n'a pas connu de réels succès. Elle demeure d'abord et avant tout un producteur et exportateur de matières premières et d'énergie. Elle a accepté des contraintes de toutes sortes pour adhérer au FMI et à la Banque mondiale, notamment l'application d'une thérapie de choc qui l'a considérablement affaiblie. Encore aujourd'hui, elle n'a pas réussi à joindre les rangs de l'OMC, ce qui fait d'elle la plus grande économie du monde à en être exclue. Le niveau de vie des Russes n'a pas encore rattrapé celui de la dernière année du régime soviétique. Bref, la Russie porte les stigmates d'un authentique processus de périphérisation et d'un processus de transition marquée par des transformations dont les effets n'ont pas été prometteurs.

La Russie porte les stigmates d'un authentique processus de périphérisation et d'un processus de transition marquée par des transformations dont les effets n'ont pas été prometteurs.

\section{BIBLIOGRAPHIE}

${ }^{1}$ Voir Michel Roche, Thérapie de choc et autoritarisme en Russie : la démocratie confisquée, Paris, L'Harmattan, 2000.
${ }^{2}$ Lynn D. Nelson et Irina Y. Kuzes, Radical Reform in Yeltsin's Russia : Political, Economic, and Social Dimensions, Armonk, NY; London, England: M.E. Sharpe, 1995, p. 44.

${ }^{3}$ Lynn D. Nelson et Irina Y. Kuzes, Radical Reform in Yeltsin's Russia : Political, Economic, and Social Dimensions, Armonk, NY; London, England: M.E. Sharpe, 1995, p. 51.

${ }^{4}$ European Bank for Reconstruction and Development (EBRD), Transition Report 2001: Energy in Transition (London : EBRD, 2001), p. 59. ${ }^{5}$ Michel Roche, «The G-7 and 'Market Reform' in Russia : 'Shock Therapy' Against Democracy», in David Mandel (ed.), The Former «State Socialist» World: Views From the Left, Montréal/New York/London: Black Rose Books, 1996, p. 129-130.

${ }^{6}$ Michel Roche, «L'adhésion de la Russie à l'OMC : les causes du retard à la fin de 2003 », Revue d'études comparatives Est-Ouest, 2003, Vol. 34, no. 2, pp. 31-52. ${ }^{7}$ Jacques Nagels, La périphérisation de l'ex-URSS?, Bruxelles, Éditions de l'Université de Bruxelles, 1993, p. 113.

${ }^{8}$ Jacques Nagels, La périphérisation de l'ex-URSS?, Bruxelles, Éditions de l'Université de Bruxelles, 1993, p. 114.

${ }^{9}$ David Mandel, Labour after Communism, Montréal/New York/London: Black Rose Books, 2004, p. 10.

${ }^{10}$ Thomas F. Remington, Politics in Russia, $2^{\text {nd }}$ Edition, 2002, p. 223.

${ }^{11}$ David Mandel, "The Working Class and the Labour Movement in Year Four of 'Shock Therapy'», in David Mandel (ed.), The Former «State Socialist» World: Views From the Left, Montréal/New York/London: Black Rose Books, 1996, p. 11-12.

${ }^{12}$ OECD Factbook 2010: Economic, Environmental and Social Statistics , (http://stats.oecd.org/Index.aspx?lang=fr). Page consultée le 30 juin 2010.

${ }^{13}$ Cécile Lefèvre, «Enfance, famille et institutions en Russie au cours des années 1990: un aperçu des évolutions récentes», Revue d'études comparatives Est-Ouest, 2003, vol. 34, no. 2, p. 84.

${ }^{14}$ Rudiger Ahrend and William Tompson, «Russia's economy: Keeping up the good times», $O E C D$ Observer, No. 249, May 2005. Page consultée le 28 juin 2010.

${ }^{15}$ Prime-TASS, 3 avril 2008, www.primetass.com/news/show.asp?topicid=68\&id=436273.

Page consultée le 29 juin 2010.

${ }^{16}$ Ben Aris, «Russia must modernise or die», www.businessneweurope.eu, 3 juin 2010.

${ }^{17} \mathrm{BBC}$ Monitoring, «Rich Russians are packed and ready to leave the country» - TV report, Center TV, 7 juin 2010. 
${ }^{18}$ Source : Федеральная служба государственой статистики, Россия в цицррах, Официальное издание, (Service de statistiques de l'État fédéral, $L a$ Russie en chiffres, publication officielle), édition 2009, p. 498.

${ }^{19}$ Wall Street Journal, 21 mars 2008.

${ }^{20}$ Reuters, 18 juin 2010.

${ }^{21}$ Interfax, 21 mai 2010.

${ }^{22}$ Maria Levitov, « Kudrin Pension Plan Attacks Putin Party, Isayev Says», Bloomberg, 1er juillet 2010.

${ }^{23}$ Programme des Nations unies pour le Développement (PNUD), Rapport mondial sur le développement humain 2009, PNUD, 2009, p. 186.

${ }^{24}$ Nezavisimaïa Gazeta, $1{ }^{\text {er }}$ juin 2010.

${ }^{25}$ Interfax, 28 mai 2010.

\section{NOTES}

${ }^{a} E n$ 1991, le PNB de l'URSS avait chuté de 13 à $15 \%$.

${ }^{b}$ En 2003, selon certaines estimations, vingt grands conglomérats contrôleraient 70 \% du PIB de la Russie.

${ }^{c}$ En 2009, la Russie est devenue le premier producteur mondial de pétrole, devant l'Arabie Saoudite.

d'emprunte cette formule au politologue Boris Kagarlitsky, qu'il a expliquée à l'occasion d'une conférence donnée à Moscou le 17 mai 2010.

${ }^{\mathrm{e}}$ En 2010, la dette publique était estimée à environ $10 \% \mathrm{du}$ PIB.

${ }^{\mathrm{f}}$ Le prix du brut de l'Oural est passé de $140 \$$ le baril en juin 2008 à environ 30 dollars en décembre de la même année.

${ }^{\mathrm{g}} \mathrm{Ce}$ projet, évalué à 3,5 milliards de dollars, serait complété en 2015.

${ }^{\mathrm{h}} \mathrm{D}$ 'après l'OCDE, l'espérance de vie des hommes est passée de 59.03 années en 2000 à 61.83 années en 2008 . Source : OECD Factbook 2010: Economic, Environmental and Social Statistics, 2010.

${ }^{i} D$ 'après le Service fédéral des Statistiques d'État, le nombre de fonctionnaires a presque doublé depuis l'arrivée au pouvoir de Poutine, passant de 866,000 en 1999 à 1.7 million en 2009. Ed Bentley, «Experts sceptical on jobs cuts plan », Moscow News, 15 juin 2010. 


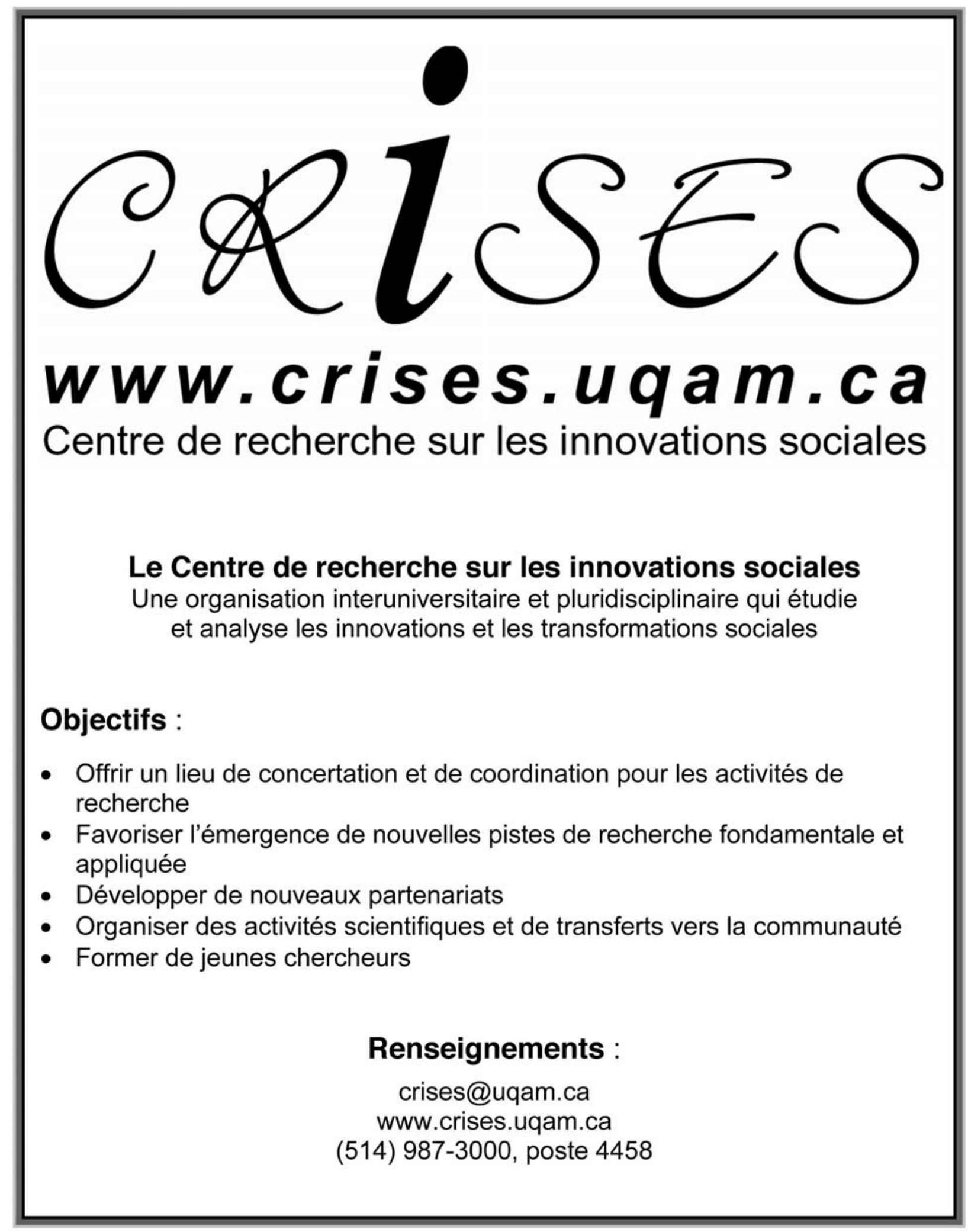

